(C) 1979 IEEE. Personal use of this material is permitted. However, permission to reprint/republish this material for advertising or promotional purposes or for creating new collective works for resale or redistribution to servers

or lists, or to reuse any copyrighted component of this work in other works must be obtained from the IEEE.

IEEE Transactions on Nuclear Science, Vol. NS-26, No. 3, June 1979

\title{
SUBNANOSECOND CHOPPER-BUNCHER SYSTEM FOR HEAVY IONS ${ }^{\star}$
}

I. Ben-Zvi, D. Bernstein, M. Birk, H. Feldman, Y. Gal and J. S. Sokolowski

Department of Nuclear Physics, The Weizmann Institute of Science, Rehovot, Israel.

\section{Summary}

A chopper-buncher for a 14U0 Pelletron is being assembled and tested. The system was designed to produce heavy ion bursts of approximately $100 \mathrm{ps}$ at 15\% transmission. This system will be used for nuclear physics fast timing experiments and can also serve as an injector to a linac. The ion source beam is sliced by a deflector-redeflector chopper operating at $1.68 \mathrm{MHz}$. The chopper is followed by a 3rd harmonic corrected buncher, which produces bunches of less than 1 ns duration at the target area, for ions up to mass 60 . Further bunching, down to 100 ps, is accomplished by a superconducting cavity operating at $430 \mathrm{MHz}$.

\section{Introduction}

The objective of the chopper-buncher described in this paper is to provide ion bursts of about $100 \mathrm{ps}$ duration at a repetition rate of a few megahertz. Such ion bursts are extremely useful in fast timing nuclear physics experiments, such as time-of-flight and lifetime measurements. This chopper-buncher can also be used to match the beam of an electrostatic accelerator to a linac booster.

Practical considerations and beam dynamics calculations led to the system layout shown in Fig. 1 . The $120 \mathrm{keV}$ ions coming from the ion source pass through the deflector-redeflector chopper ${ }^{1}$. A conventional single deflector chopper would have introduced a large energy spread causing a significant degradation in the performance of the buncher which follows it. The double deflection scheme used reduces this energy spread by an order of magnitude. The third harmonic component added to the basic sine wayeform driving the buncher considerably improves the bunching operation. In order to reduce the broadening of the bunch due to the ion energy spread, originating mostly in the ion source, the buncher is placed close to the acceleration tube. Beam dynamics calculations have shown ${ }^{2}$ that this system will produce bunches of approximately 0.6 ns FWHM at the target area for ions up to mass 60 . Further bunching is done by a superconducting cavity ${ }^{3}$ operating at $430 \mathrm{MHz}$. Accord. ing to the numeric calculations done, bunches of $20 \mathrm{ps}$ FWHM should be achieved. The preacceleration chopper and buncher operate at $1.68 \mathrm{MHz}$ and $3.36 \mathrm{MHz}$, respectively. These frequencies are obtained by down counting a signal sampled from the superconducting cavity. An electronic phase shifter is used to correct for fluctuations in the time of arrival of the $1 \mathrm{~ns}$ bunches at the target area. These fluctuations are mainly caused by variations in the terminal voltage of the accelerator. The arrival time is measured by a beam pick-up placed before the switching magnet.

The system described has been constructed and is now being assembled and tested.

This work was supported by a grant from the United States-Israel Binational Science Foundation (BSF) Jerusalem, Israel.

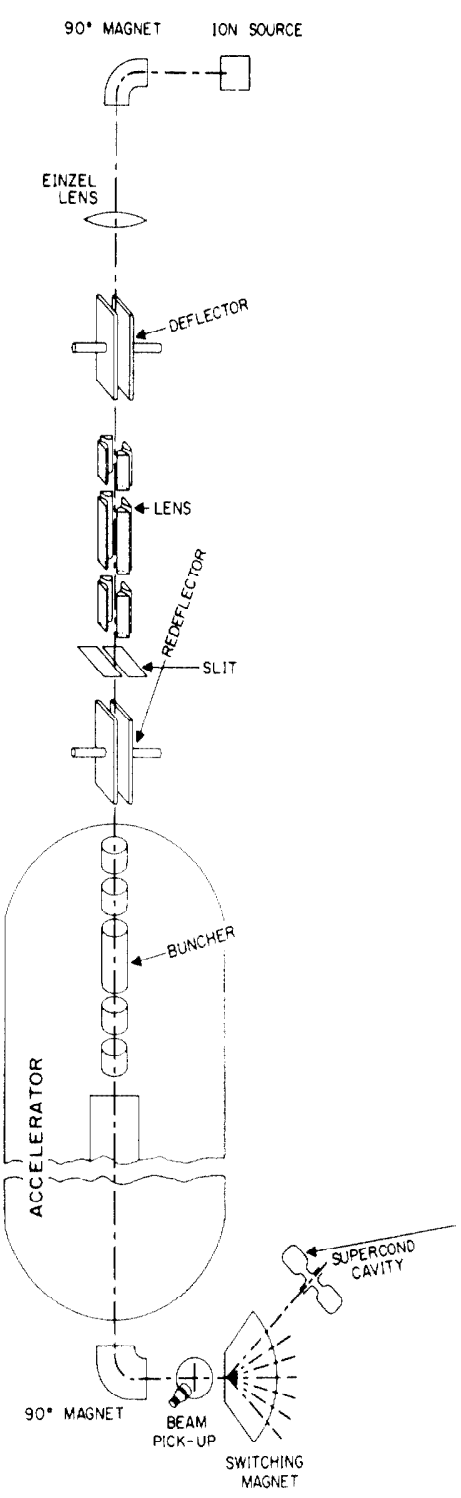

Fig. 1. Chopper-buncher system layout.

\section{Preacceleration Chopper-Buncher}

As pointed out by Fowler and Good'4, a simple chopper consisting of a deflector followed by a slit produces an energy spread, $\Delta E$, given by

$$
\Delta E \cdot \Delta t \geq y \cdot p_{y}
$$

where $\Delta t$ is the duration of a beam chop, $y$ is the beam spread in the transverse coordinate, and $p_{y}$ is the transverse momentum spread. This energy spread may seriously deteriorate the performance of a buncher following such a chopper. The deflector-redeflector chopper shown in Fig. I solves this problem, since the 
redeflector compensates the energy spread introduced by the deflector. An electrostatic quadrupole triplet lens of focal length $0.5 \mathrm{~m}$ is situated midway between the deflector and redeflector, the separation of which is $2 \mathrm{~m}$. The magnification of this lens from deflector to redeflector is -1 . Thus, a particle passing off the center line at the deflector will pass off the center line on the opposite side at the redeflector. Hence, the energy modulation caused by the fringing fields of the deflector will be cancelled out by those of the redeflector, provided that both, deflector and redeflector, act on the particle with the same field and phase. The deflection plates are $12 \mathrm{~cm}$ long, $5 \mathrm{~cm}$ wide and the plate separation is $3 \mathrm{~cm}$. Under typical operating conditions, $1.5 \mathrm{kV}$ rms across the plates will produce the required deflection for ions up to about mass 60 . Detailed calculations ${ }^{2}$ taking into account the beam emittance, the fringing fields of the deflectors and lens aberrations gave $\Delta E \cdot \Delta t=0.2 y \cdot p_{y}$.

The preacceleration buncher is $35 \mathrm{~cm}$ long and consists of five $4 \mathrm{~cm}$ ID tube sections. The separation between adjacent sections is approximately $2.5 \mathrm{~cm}$. The two end sections are at ground potential. Each of the remaining three sections is either grounded or connected to the RF source via high voltage RF relays, depending on the velocity of the ions to be bunched. The buncher is located $60 \mathrm{~cm}$ before the acceleration tube. The RF voltages required are typically $5 \mathrm{kV}$ rms at $3.36 \mathrm{MHz}$ and $250 \mathrm{~V}$ rms at $10.08 \mathrm{MHz}$, which is the corrective third harmonic superimposed upon the basic frequency.

Figure 2 shows the calculated ${ }^{2}$ time distribution of mass 60 ions with $14 \mathrm{MV}$ on the terminal and $2 \mu \mathrm{g} / \mathrm{cm}^{2}$ carbon foil stripper, at $14 \%$ beam transmission.

182 MEV MASS 60 IONS

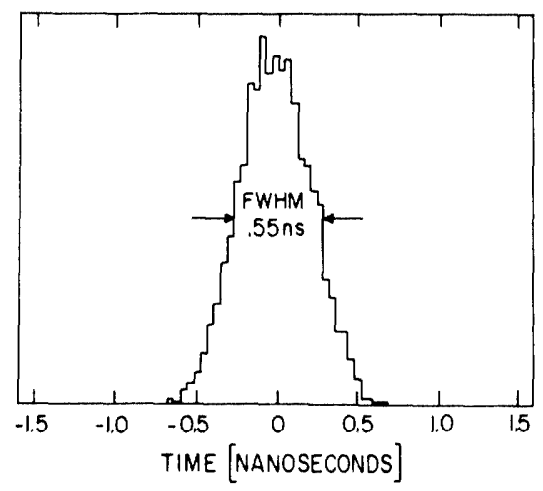

Fig. 2. Computer calculated distribution of 6370 particles past the analyzing magnet.

\section{Postacceleration Superconducting Buncher}

In order to obtain sub-100 picosecond bunches at the target, further bunching of the $1 \mathrm{~ns}$ ion bursts is required. Since the energy spread of the beam introduces time spread, this additional fast buncher has to be located close to the target. Hence, the fast buncher requires strong electric fields of the order of $10 \mathrm{MV} / \mathrm{m}$. Achieving such high fields in a normal conducting cavity necessitates hundreds of kilowatts of RF power. In a superconducting cavity such fields can be easily reached with only a few watts of RF power.

The superconducting cavity used for the fast bunching ${ }^{5}$ was constructed at Stanford University in
182 MEV MASS 60 IONS

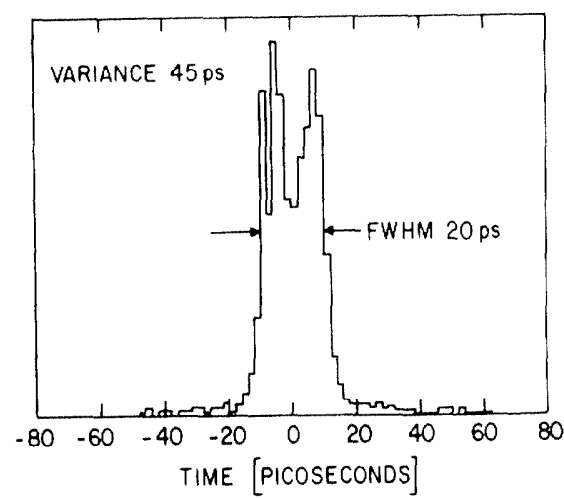

Fig. 3. Computer calculated distribution of 6370 particles at the target.

cooperation with the Weizmann Institute. It is a niobium reentrant cavity, $35 \mathrm{~cm}$ in diameter, $10 \mathrm{~cm}$ long and it has an acceleration gap of $2 \mathrm{~cm}$. The natural resonant frequency of this cavity is approximately $430 \mathrm{MHz}$. Electric fields of $12 \mathrm{MV} / \mathrm{m}$ are readily obtained with this cavity, at $Q_{0}$ values of approximately $1 \times 10^{9}$.

As earlier mentioned, the synchronization of the injection into the superconducting cavity is accomplished by downcounting the $430 \mathrm{MHz}$, to drive the preacceleration chopper-buncher, and by a beam pick-up measuring the time of arrival of the bunches. This beam pick-up consists of a $25 \mu \mathrm{m}$ tungsten wire, which emits electrons when hit by the beam. A negative $3 \mathrm{kV}$ potential is applied to the wire in order to accelerate the electrons radially. Some of these electrons are detected by a microchannel plate electron multiplier, which is located at a back angle of $60^{\circ}$.

Figure 3 shows the calculated time distribution of the ion bunch of Fig. 2 after further bunching by the superconducting cavity.

\section{Electronics}

The block diagram of the electronics set-up is shown in Fig. 4. The superconducting cavity is driven to free run at its resonant frequency of $430 \mathrm{MHz}$ The Cavity Control linit described in references 5 and 6 serves as the cavity driver. A signal sampled from the cavity is downcounted to $3.36 \mathrm{MHz}$ by a temperature controlled scaler employing fast flip-flops (MC 1690L). The time jitter of the scaler output is less than 20 ps. The $3.36 \mathrm{MHz}$ signal passes through a phase shifter, which syncronizes the time of arrival of the ion bunches to the phase of the superconducting cavity. The beam pick-up provides "start" pulses to a time-to-amplitude converter (TAC), which gets "stop" pulses from the frequency divider. The output of the TAC controls the phase shifter, employing the peak stabilization technique used in nuclear spectroscopy. The phase shifting circuit is based upon a varactor diode in parallel with a resonant circuit. 


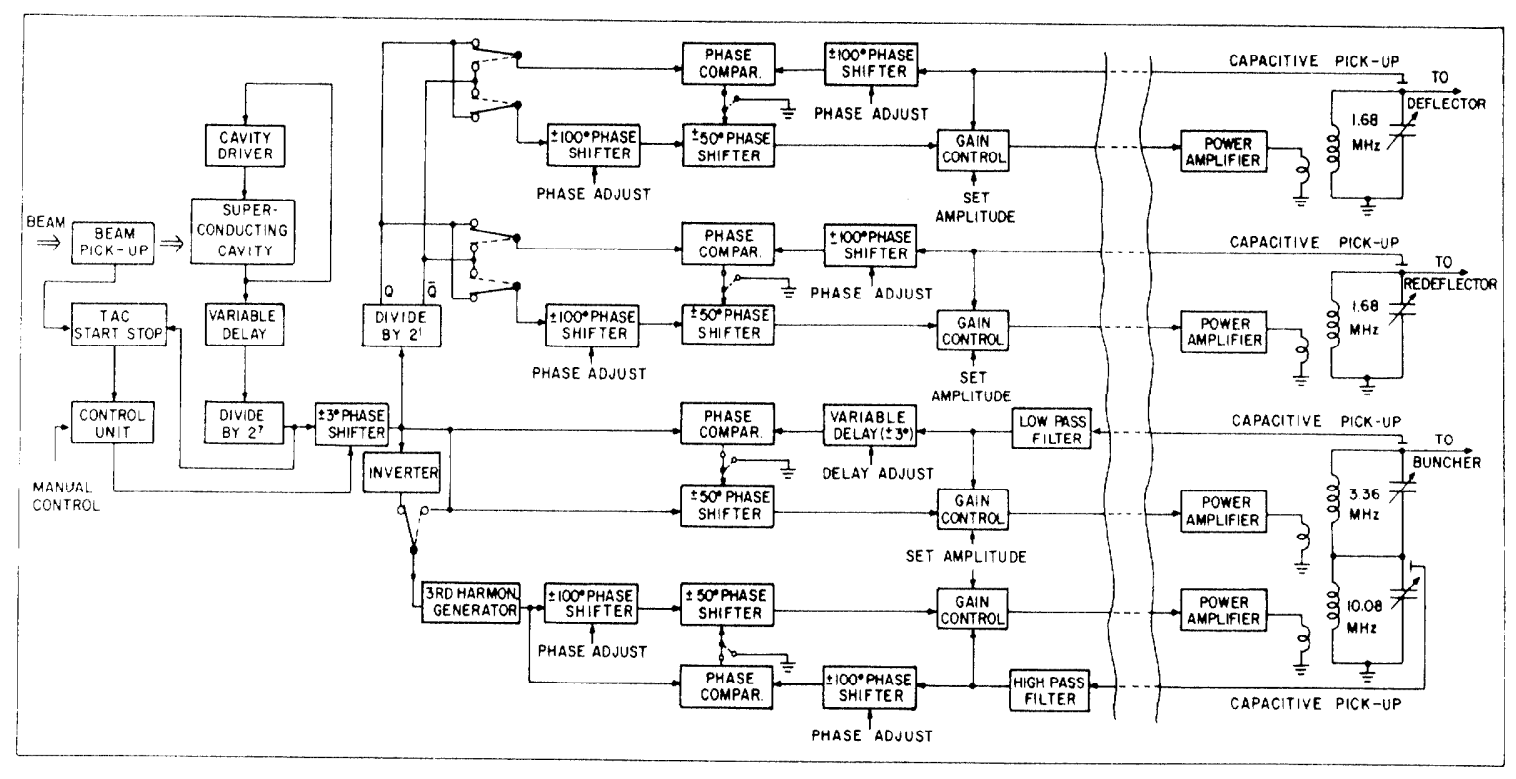

Fig. 4. Electronics block diagram.

The $3.36 \mathrm{MHz}$ phase shifter output drives the buncher branch (basic frequency and third harmonic) and, through an additional flip-flop, the chopper branch (deflector and redeflector). Any required phase between the $430 \mathrm{MHz}$ signal and the $3.36 \mathrm{MHz}$ buncher signal can be set by the variable delay and the manual control of the $\pm 3^{\circ}$ phase shifter. The phases of the third harmonic and of the two deflectors are set, with respect to the buncher main frequency, by $180^{\circ}$ switching and by $\pm 100^{\circ}$ electronic phase shifters. Double balanced mixers compare the phases of reference signals to the respective sampled signals and control the $\pm 50^{\circ}$ phase shifters, in order to correct for phase drifts and fluctuations. These electronic RC phase shifters employ field effect transistors as variable resistors. The sampled signals coming from the capacitive pick-ups are peak-detected and their amplitudes are compared to manually set voltages. The voltage comparators control the gain of operational amplifiers in the drive path. The gain control is accomplished by the use of a field effect transistor as a variable resistor in the negative feedback network. The electric fields of the chopper-buncher elements are thus set and stabilized. The measured amplitude noise is typically $\pm 0.1 \%$ and the phase noise is typically $+0.1^{0}$.

\section{References}

1. I. Ben-Zvi, "Beam Dynamics for a Cryogenic Booster Linac", Particle Accelerators 8, pp.31-41 (1977).

2. I. Ben-zvi and Z. Segalov, "Beam Dynamics of a Linac Injection System", Particle Accelerators (in press).

3. P.H. Ceperley, I. Ben-Zvi, H.F. Glavish and S.S. Hanna, "Superconducting Re-entrant Cavities for Heavy Ion Linacs", IEEE Trans. Nuc1.Sci. NS-22, No. 3, pp. 1153-1156 (1975).

4. T.K. Fowler and W.M. Good, "A Theory on Obtaining Short Bursts of Ions from a Beaml of Ions", Nucl. Instr, and Meth. 7, pp. 245-252 (1960).

5. P.H. Ceperley, H.F. Glavish, S.S. Hanna, M. Samuel and J.S. Sokolowski, "Beam Tests and Phase Locking of Superconducting Cavities for Heavy Ion Linac", IEEE Trans. Magnetics MAG-13, No. 1, pp. 520-523 (1977).

6. J.S. Sokolowski, P.H. Ceperley, M. Samuel, M.Birk, H.F. Glavish and S.S. Hanna, "Status Report on Stanford's Superconducting Heavy Ion Linac Project", IEEE Trans. Nucl. Sci. NS-24, No. 3, pp. 1141-1143, (1977). 